\title{
Modeling and Flocking Consensus Analysis for Large-Scale UAV Swarms
}

\author{
Li Bing, Li Jie, and Huang KeWei \\ School of Mechatronical Engineering, Beijing Institute of Technology, Beijing 100081, China \\ Correspondence should be addressed to Huang KeWei; binlin_lee@163.com
}

Received 31 July 2013; Accepted 7 October 2013

Academic Editor: J. A. Tenreiro Machado

Copyright ( 2013 Li Bing et al. This is an open access article distributed under the Creative Commons Attribution License, which permits unrestricted use, distribution, and reproduction in any medium, provided the original work is properly cited.

\begin{abstract}
Recently, distributed coordination control of the unmanned aerial vehicle (UAV) swarms has been a particularly active topic in intelligent system field. In this paper, through understanding the emergent mechanism of the complex system, further research on the flocking and the dynamic characteristic of UAV swarms will be given. Firstly, this paper analyzes the current researches and existent problems of UAV swarms. Afterwards, by the theory of stochastic process and supplemented variables, a differentialintegral model is established, converting the system model into Volterra integral equation. The existence and uniqueness of the solution of the system are discussed. Then the flocking control law is given based on artificial potential with system consensus. At last, we analyze the stability of the proposed flocking control algorithm based on the Lyapunov approach and prove that the system in a limited time can converge to the consensus direction of the velocity. Simulation results are provided to verify the conclusion.
\end{abstract}

\section{Introduction}

UAV is an advanced system with high autonomy for intelligent combat [1]. In the future, UAVs will be used for complex tasks, such as surveillance, reconnaissance, and precision strike missions. Many organizations have foreseen that in the near future, swarms of UAVs will replace single ones for more complicated missions in more uncertain and possibly hostile environments [2]. Therefore, many researchers are studying groups of cooperative UAVs.

(A) Related Work on the UAV Swarms Problem. The new challenges imposed by UAV swarms have attracted many researchers. New control mechanisms, application domains, simulation models, and simulation tools have been developed to tackle issues in different aspects of the swarm. Currently, a completely new topic is opening up in the area of UAV swarms performing different missions cooperatively. [3], the method of evolutionary pinning control is applied to UAV swarms successfully. Path planning and routing are investigated in $[4,5]$, using multiobjective evolutionary algorithm. The path planning problem in three-dimensional environment without any obstacles is addressed in $[6,7]$ and with only static obstacles in [8]. [9], cooperative searching problem is discussed for the purpose of detecting moving and evading targets in a hazardous environment. A similar cooperative searching problem is also discussed in $[10,11]$. Reference [12] investigates the automatic target recognition (ATR) problem in UAV control and proposes a distributed strategy for UAV swarms. Task allocation problem is discussed in [13-18] using different methodologies. Some applications of using a UAV swarm to search and destroy targets could be found in [1922]. [23], a swarm simulator for target searching is implemented with Java. Garcia introduces a multi-UAVs simulator implemented with X-Plane-a commercial flight simulator [24]. Russell et al. present a parallel swarm simulation environment which utilizes an existing parallel emulation and simulation tool called SPEEDS [25]. MASON [26] is a general purpose multiagents simulation library utilized in our previous work, along with MATLAB based UAV simulator [27].

(B) Related Work on the Consensus Flocking Problem. Most research on formation of agent swarms uses distributed techniques by Reynolds' seminal work on the mobility of flocks [28], which prescribes three fundamental operations for each robot to realize distributed flocking-separation, alignment, and cohesion $[29,30]$. One of the earliest attempts to realize 
flocking through a set of basic behaviors including safe wandering, aggregation, dispersion, and homing to implement flocking is by Mataric [31]. Kelley and Keating realize flocking with robots based on leader-following behavior [32]. Hayes and Dormiani-Tabatabaei [33] propose a flocking algorithm based on two behaviors: collision avoidance and velocity-matching flock centering. Holland et al. [34] propose a flocking algorithm for UAV similar to Reynolds. A host is used as an intermediate station for receiving each UAV's range, bearing, and velocity and sending them to other UAVs to simulate the sensing process of one UAV for perceiving range, bearing, and heading of its neighbors. Ferrante et al. [35] introduce a new communication strategy called the information aware communication for alignment behavior. Recently, Stranieri et al. [36] perform flocking with a swarm of behaviorally heterogeneous mobile robots.

In this paper, we consider models for flocking swarms. Firstly, a mathematical model of cooperative system is established by using Markov stochastic process and calculus analysis. Then, the control law for UAV swarm is established based on artificial potential field. At last, we analyze the stability of the proposed flocking control algorithm based on the Lyapunov approach and prove the conclusion that the system in a limited time can converge to the consensus direction of the velocity. Simulation results are provided to verify the conclusion.

\section{The Model of the UAVs Swarms}

2.1. Differential Integral Model. Let $C(t)$ denote the state of the UAV swarms at time $t ; C(t)=0$ identifies the state that UAV swarms are stable at time $t$. The state of UAV $i$ at time $t$ is denoted by $c_{i}(t)=\left(p_{i}(t), o_{i}(t)\right)$, in which the first element $p_{i}(t)=\left(x_{i}(t), y_{i}(t), z_{i}(t)\right)$ is the UAV's position in the environment at time $t$, and the second element $o_{i}(t)$ is the UAV's orientation. The UAV's dynamics is subject to its physical curvature radius constraints, leading to the fact that it can only change its orientation by at most one step, which is described as go straight, go up, go down, turn left, turn upper left, turn lower left, turn right, turn upper right, and turn lower right.

In order to obtain Markov random process, the new state of process is derived by supplement of variable [37,38], which is described as follows:

$$
\begin{gathered}
P_{i}(t)=P(C(t)=i), \quad i=0,1,2, \ldots, N, \\
P_{j k}(x, t)=P\left(C(t)=j, x<y_{j, k}(t)<x+d x\right), \\
j=N+1, \ldots, M, k=0,1,2, \ldots, N,
\end{gathered}
$$

where $y_{i}(t)$ is the dwell time after state $i$. So it is easy to verify that $\left\{U(t), 0 \leq t \leq T_{0}\right\}=(C(t), y(t))$ is a broad Markov random process.
The probability of state transition after $\Delta t$ can be obtained using total probability theorem:

$$
\begin{aligned}
P_{0}(t+\Delta t)= & \left(\sum_{i=0}^{M} P(C(t)=i, C(t+\Delta t)=i)\right) \\
= & P_{0}(t)\left(1-\sum_{i=1}^{N} \lambda_{0 i} \Delta t+o(\Delta t)\right)+\sum_{i=1}^{N} \lambda_{i 0} P_{k}(t) \Delta t \\
& +\sum_{j=N+1}^{M} \int_{0}^{T} P_{j}(x, t) r_{j}(x) \Delta t d x+o(\Delta t) .
\end{aligned}
$$

According to (2) we can get the all probability:

$$
\begin{gathered}
P_{k}(t+\Delta t)=P_{k}(t)\left(1-\sum_{i=1, i \neq k}^{N} \lambda_{k i} \Delta t+o(\Delta t)\right) \\
+\sum_{i=1, i \neq k}^{N} \lambda_{i k} P_{k}(t) \Delta t+o(\Delta t), \\
\quad k=1,2, \ldots, N, \\
P_{j}(x, t)=P(C(t)=j ;
\end{gathered}
$$

the time of self-organised $C_{\text {system }}$ is $x$,

$$
\begin{gathered}
C(t+\Delta t)=j) \\
=P_{j}(x, t)\left(1-r_{j}(x) \Delta t+o(\Delta t)\right), \\
j=N+1, \ldots, M,
\end{gathered}
$$

where $\lambda_{i j}$ is the average sustained rate of each state and $r_{j}(x)$ is the average repair rate at state $j$. Similarly, the expression of state transition rate for $P_{j}(x+\Delta x, t+\Delta t)$ can be derivated.

Differentiate the expression for state transition probability to derive its limit. Then the mathematical model can be described using integral-differential equations as follows:

$$
\begin{aligned}
& \frac{d P_{0}(t)}{d t}+\sum_{i=0, i \neq k}^{N} \lambda_{0 k} P_{0}(t) \\
& =\sum_{i=0, i \neq k}^{N} \lambda_{i 0} P_{i}(t) \\
& +\sum_{j=N+1}^{M} \int_{0}^{T} r_{j}(x) p_{j}(x, t) d x \\
& \frac{d P_{k}(t)}{d t}+\sum_{i=0, i \neq k}^{N} \lambda_{k i} P_{k}(t) \\
& =\sum_{i=0, i \neq k}^{N} \lambda_{i k} P_{i}(t), \\
& k=1,2, \ldots, N,
\end{aligned}
$$




$$
\begin{array}{r}
\frac{\partial p_{j}(x, t)}{\partial x}+\frac{\partial p_{j}(x, t)}{\partial t}+r_{j}(x) p_{j}(x, t)=0, \\
j=N+1, \ldots, M .
\end{array}
$$

The boundary and initial conditions are

$$
\begin{gathered}
P_{0}(0)=1, \quad P_{1}(0)=0, \ldots, P_{i}(0)=0, \ldots, P_{N}(0)=0, \\
P_{j}(0, t)=\sum_{i=0}^{N} \lambda_{i j} P_{i}(t), \quad j=N+1, \ldots, M .
\end{gathered}
$$

Theorem 1. The reliability of coordination system has uniqueness and nonnegative solution on $C[0, T]$.

Proof. According to the initial conditions we can get the analytic solution of the partial differential equation $[39,40]$.

Set

$$
\begin{gathered}
a_{0}=\sum_{i=1}^{N} \lambda_{0 i}, \ldots, a_{j}=\sum_{i=1}^{N} \lambda_{j i}, \ldots, a_{N}=\sum_{i=1}^{N} \lambda_{N i}, \\
P_{j}(x, t)=P_{i}(0, t-x) \exp \left(-\int_{0}^{x} r_{i}(\mu) d \mu\right), \\
\frac{d P_{0}(t)}{d t}=-a_{0} P_{0}(t)+\sum_{i=0, i \neq k}^{N} \lambda_{i 0} P_{i}(t) \\
+\sum_{j=N+1}^{M} \int_{0}^{T} P_{j}(0, t-x) \exp \left(-\int_{0}^{x} r_{i}(\mu) d \mu\right) \\
\times \int_{0}^{T_{0}} \sum_{i=N+1}^{M} \int_{0}^{s} P_{i}(0, \tau) \exp \left(-\int_{0}^{s-\tau} r_{i}(\mu) d \mu\right) \\
+\exp \left(-a_{0} t\right)+\sum_{i=1}^{N} \lambda_{i 0} \int_{0}^{T_{0}} P_{i}(s) \exp \left(-a_{0}(t-s)\right) d s \\
\left.\times r_{i}(s-\tau) d \tau\right]
\end{gathered}
$$

$$
\begin{aligned}
& \times \exp \left(-a_{0}(t-s)\right) d s \\
= & \exp \left(-a_{0} t\right)+\sum_{i=1}^{N} \lambda_{i 0} \int_{0}^{T_{0}} P_{i}(s) \exp \left(-a_{0}(t-s)\right) \times d s \\
& +\sum_{i=N+1}^{M} \int_{0}^{T_{0}} P_{i}(0, \tau) d \tau \\
& \times \int_{0}^{T_{0}-\tau} \exp \left(-a_{0}\left(T_{0}-\tau\right)+a_{0} v\right. \\
& \left.\quad-\int_{0}^{v} r_{i}(\mu) d \mu\right) r_{i}(v) d v \\
= & \exp \left(-a_{0} t\right)+\sum_{i=1}^{N} \lambda_{i 0} \int_{0}^{T_{0}} P_{i}(s) k_{0} d s \\
& +\sum_{i=N+1}^{M} \int_{0}^{T_{0}} P_{i}(0, \tau) K_{i}(t-\tau) d \tau,
\end{aligned}
$$

where

$$
\begin{gathered}
k_{0}=\exp \left(-a_{0}(T-s)\right) \\
k_{i}(T-\tau)=\int_{0}^{T-\tau} k_{0} * \exp \left(a_{0} v-\int_{0}^{v} r_{i}(\mu) d \mu\right) r_{i}(v) d v, \\
i=N+1, \ldots, M, \\
P_{j}(t)=\sum_{i=0, i \neq j}^{N} \int_{0}^{T} \exp \left(-a_{j}(T-s)\right) \lambda_{i j} P_{i}(s) d s \\
=\sum_{i=0, i \neq j}^{N} \int_{0}^{T} k_{j} \lambda_{i j} P_{i}(s) d s, \quad j=1,2, \ldots, N,
\end{gathered}
$$

where $k_{j}=\exp \left(-a_{j}(T-s)\right), j=1,2, \ldots, N$.

So we can get the following equation:

$$
\begin{aligned}
& P_{j}(0, t)= \sum_{i=0}^{N} \lambda_{i j} \sum_{l=0, l \neq j}^{N} \int_{0}^{T} k_{j} \lambda_{l j} P_{l}(s) d s \\
&=\int_{0}^{T} k_{j}\left(\sum_{i=0}^{N} \lambda_{i j}\right)\left(\sum_{l=0, l \neq j}^{N} \lambda_{l j} P_{l}(s)\right) d s, \\
& \quad j=N+1, \ldots, M .
\end{aligned}
$$

Assuming

$$
\begin{aligned}
P(t) & =\left(P_{0}(t), P_{1}(t), \ldots, P_{N}(t), P_{N+1}(0, t), \ldots, P_{M}(0, t)\right), \\
f(t) & =\left(f_{0}(t), f_{1}(t), \ldots, f_{N}(t), f_{N+1}(0, t), \ldots, f_{M}(0, t)\right) \\
& =\left(\exp \left(-a_{0} t\right), 0, \ldots, 0, \ldots, 0\right),
\end{aligned}
$$


then, the solution of the system can be converted into vectors format as follows:

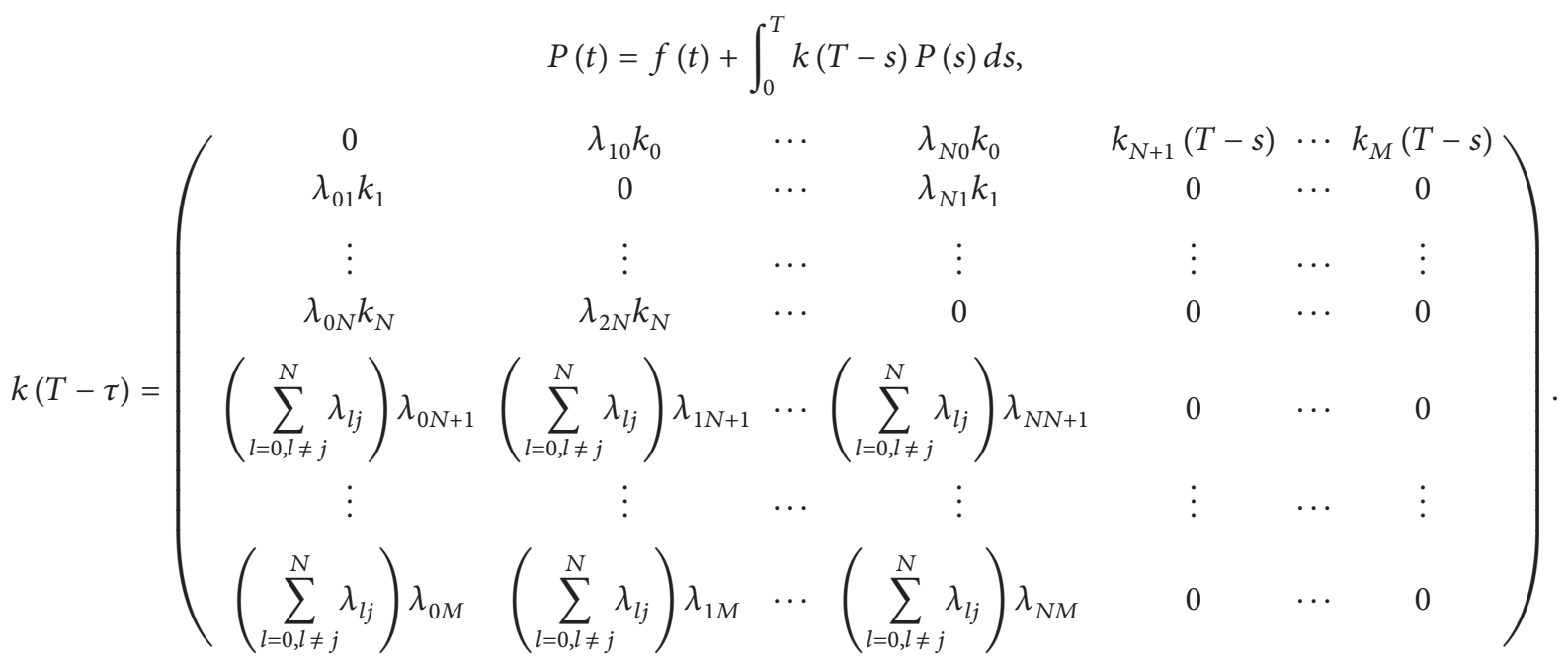

Any component of $F(t)$ and $G(t-h)$ vector is nonnegative. The functions $F_{j}(t)$ and $G_{j}(t-h), j=1,2, \ldots, M$ are limitary on the domain $0<T<+\infty$. The solution of integral equation is unique and nonnegative on $C[0, T]$. So the reliability of coordination system has unique and nonnegative solution on $C[0, T]$.

2.2. Probabilistic Analysis Based on State Transformation. The behavior evolution of the UAV swarm system is a limited Markov decision process. Suppose that the probability distribution of the system state is $P(N, t)$ at time $t$. Then at time $t+\tau$, the probability distribution is $P(N, t+\tau)$. According to the relationship of the probability density at different time, the marginal probability density $P(N, t+\tau)$ is $P(N, t+\tau)=$ $\int P(\eta, t) P(N, t+\tau \mid \eta, t) d \eta$.

And the time derivative of the $P(N, t+\tau)$ is

$$
\frac{d P(N, t)}{d t}=\lim _{\tau \rightarrow 0} \frac{P(N, t+\tau)-P(N, t)}{\tau} .
$$

Define $W_{t}(\eta, N)$ as the transition probability density from state $\eta$ to state $N$ in unit time during time interval $[t, t+\tau]$. So the transition probability from state $\eta$ to state $N$ during time interval $[t, t+\tau]$ is $\tau W_{t}(\eta, N)$. Then the probability by which the transition does not happen is

$$
1-\tau \int W_{t}(\eta, N) \delta(N-\eta) d n
$$

where $\delta(N-\eta)=P(N, t+\tau \mid \eta, t)$, when $\tau=0$. Thus,

$$
\frac{d P(N, t)}{d t}=\int\{W(\eta, n) P(\eta, t)-W(n, \eta) P(n, t)\} d \eta \text {. }
$$

Equation (13) describes the evolution of the system states over time, which is the primary equation model of the UAV swarms behavior.

\section{Flocking Control of UAV Swarms}

3.1. Flocking Control Law. In this section, first we design a distributed flocking control law. Assuming that each UAV senses its own position and velocity and is able to obtain its neighbors' position and velocity, the UAV swarms form flocking behaviour model structure control law as follows:

$$
\begin{aligned}
U_{i}= & \text { Uniformity }(v) \sum_{j=1, j \neq i}^{N} f\left(p_{i, j}\right)\left(p_{i}-p_{j}\right) \\
& +\beta\left(p_{i}-p_{\text {goal }}\right)+\sum_{j=1, j \neq i}^{N} a_{i, j}\left(p_{i, j}\right)\left(v_{j}-v_{i}\right),
\end{aligned}
$$

where Uniformity $(v)=\left(\alpha \sum_{i \neq j}\left\|v_{i}-v_{j}\right\|_{2}\right)$. Note that alignment at a common velocity is equivalent to Uniformity $(v)=$ 0. $P_{i, j}$ is the distance between the individual $i$ and $j . U_{i j}$ is potential function and satisfies the condition $[29,30]$ :

(i) $U_{i R}\left(\left\|p_{i}-p_{j}\right\|\right) \rightarrow \infty,\left\|p_{i}-p_{j}\right\|<R_{\min }$,

(ii) $R_{\min } \leq\left\|p_{i}-p_{j}\right\| \leq R_{\max }, \exists ! \min U_{i R}$.

3.2. Stability Analysis. Consider the following positive semidefinite function:

$$
E=\frac{1}{2}\left(\sum_{i=1}^{N}\left(\sum_{j=1, j \neq i}^{N} U_{i, j}+K_{i} U_{i, \text { goal }}\right)+v_{i}^{T} v_{i}\right) .
$$


In order to facilitate writing, we simplify the certification process variable substitution as follows:

$$
\begin{aligned}
& \bar{U}_{i}=\left(\sum_{j=1, j \neq i}^{N} U_{i, j}+K_{i} U_{i, \text { goal }}\right), \\
& \dot{E}=\frac{1}{2} \sum_{i=1}^{N} \dot{\bar{U}}_{i}-\sum_{i=1}^{N} v_{i}^{T} U_{i} \\
& =\frac{1}{2} \sum_{i=1}^{N} \dot{\bar{U}}_{i}+\sum_{i=1}^{N} v_{i}^{T}\left(-\sum_{j=1, j \neq i}^{N} \nabla_{p_{i}} U_{i j}\left(p_{i, j}\right)-K_{i} \nabla_{p_{i}} U_{i A}\left(p_{i, j}\right)\right. \\
& \left.-\sum_{j=1, j \neq i}^{N} \alpha_{i, j}\left(v_{i}-v_{j}\right)\right) \\
& =\sum_{i=1}^{N} v_{i}^{T} \nabla_{p_{i}} \bar{U}_{i}+\sum_{i=1}^{N} v_{i}^{T}\left(-\sum_{j=1, j \neq i}^{N} \nabla_{p_{i}} U_{i j}\left(p_{i, j}\right)\right. \\
& -K_{i} \nabla_{p_{i}} U_{i, \text { goal }}\left(p_{i, \text { goal }}\right) \\
& \left.-\sum_{j=1, j \neq i}^{N} \alpha_{i, j}\left(v_{i}-v_{j}\right)\right) \\
& =\sum_{i=1}^{N} v_{i}^{T} \nabla_{p_{i}}\left(\sum_{j=1, j \neq i}^{N} U_{i, j}+K_{i} U_{i, \text { goal }}\right) \\
& +\sum_{i=1}^{N} v_{i}^{T}\left(-\sum_{j=1, j \neq i}^{N} \nabla_{p_{i}} U_{i j}-K_{i} \nabla_{p_{i}} U_{i, \text { goal }}\right. \\
& \left.-\sum_{j=1, j \neq i}^{N} \alpha_{i, j}\left(v_{i}-v_{j}\right)\right) \\
& =\sum_{i=1}^{N} v_{i}^{T}\left(-\sum_{j=1, j \neq i}^{N} \alpha_{i, j}\left(v_{i}-v_{j}\right)\right) \\
& =-v^{T}\left(L_{C} \otimes I_{3}\right) v,
\end{aligned}
$$

where $L_{c}$ is UAV swarms system satisfying the Laplacian matrix of the communication conditions. Therefore, the quadratic form is explicitly described as follows:

$$
\dot{E}=-v_{x}^{T} L_{c} v_{x}-v_{y}^{T} L_{c} v_{y}-v_{z}^{T} L_{c} v_{z} \leq 0 .
$$

Consider the following collections: $\left\{v_{i}, P_{i, j} \mid E \leq C\right\}$ is a closed set. The following is to verify that it is a compact set, and there is a clear conclusion that $P_{i, j} \leq C$. Similarly $v_{i}^{T} v_{i} \leq C,\left\|v_{i}\right\| \leq C^{1 / 2}$, and according to the definition of the potential field we obtain $\left\|P_{i, j}\right\| \leq U_{i, j}^{-1}(C(N-1))$. According to the LaSalle invariance principle, the system will converge to the largest invariant set in the area and meet $\dot{E}=0$. According to $\dot{E}=0$, when the system enters the steady state, the speed of each individual is equal, and all individuals move to the target position $P_{\text {goal }}$, making the overall potential energy minimum.
Theorem 2. Consider the UAV swarms consisting of $N$ UAVs. The position of individual $i$ is $p_{i}$. All individuals in the swarms will eventually build up to the spherical region:

$$
\left\|p_{i}-p_{c}\right\| \leq \frac{2 \lambda_{\max }(G)(N-1) l C}{\lambda_{\min }\left(A^{T} G+G A\right)} .
$$

Proof. Consider

$$
\begin{gathered}
\dot{P}_{c}=V_{c} \\
\dot{V}_{c}=\frac{1}{N} \sum_{i=1}^{N} \dot{v}_{i}=\frac{1}{N} \sum_{i=1}^{N}\left(-\sum_{j=1, j \neq i}^{N} \nabla_{p_{i}} U_{i j}\left(\left\|p_{i}-p_{j}\right\|\right)\right) \\
-\beta_{i} \nabla_{p_{i}} U_{i A}\left(\left\|p_{i}-p_{c}\right\|\right)-\sum_{j=1, j \neq i}^{N} \alpha_{i, j}\left(v_{i}-v_{j}\right)=0,
\end{gathered}
$$

where $P_{c}(t)=(1) / N \sum_{i=1}^{N} p_{i}(t), V_{c}=(1) / N \sum_{i=1}^{N} \alpha_{i, j} v_{i}$.

By making the variable replacement $\varepsilon_{i p}=P_{i}-P_{c}, \varepsilon_{i p}=$ $v_{i}-v_{c}$, we get

$$
\begin{gathered}
\dot{\varepsilon}_{i p}=\varepsilon_{i v}, \\
\dot{\varepsilon}_{i p}=\left(-\sum_{j=1, j \neq i}^{N} \nabla_{p_{i}} U_{i j}\left(\left\|p_{i}-p_{j}\right\|\right)\right. \\
\left.-\beta_{i} \nabla_{p_{i}} U_{i A}\left(\left\|p_{i}-p_{c}\right\|\right) \sum_{j=1, j \neq i}^{N} \alpha_{i, j}\left(v_{i}-v_{j}\right)\right) \\
=-\beta_{i} \varepsilon_{i p}-N\left(\sum_{j=1, j \neq i}^{N} \alpha_{i, j}\right) \varepsilon_{i v} \\
-\sum_{j=1, j \neq i}^{N} \nabla_{p_{i}} U_{i j}\left(\left\|p_{i}-p_{j}\right\|\right) .
\end{gathered}
$$

Then

$$
\begin{gathered}
\dot{\zeta}_{i}=\left(\begin{array}{c}
\dot{\varepsilon}_{i p} \\
\dot{\varepsilon}_{i v}
\end{array}\right)=\left(\begin{array}{c}
0 \\
\left.-\beta_{i}-N\left(\sum_{j=1, j \neq i}^{N} \alpha_{i, j}\right)\right) \zeta_{i} \\
-\left(\sum_{j=1, j \neq i}^{N} \nabla_{p_{i}} U_{i j}\left(\left\|p_{i}-p_{j}\right\|\right)\right), \\
\left.A=\left(\begin{array}{c}
0 \\
-\beta_{i}-N\left(\sum_{j=1, j \neq i}^{N} \alpha_{i, j}\right.
\end{array}\right)\right), \\
B=\left(\sum_{j=1, j \neq i}^{N} \nabla_{p_{i}} U_{i j}\left(\left\|p_{i}-p_{j}\right\|\right)\right.
\end{array}\right) .
\end{gathered}
$$


Since $K_{i}, N>0$,

$$
\begin{aligned}
& \lambda(A)= \frac{-N\left(\sum_{j=1, j \neq i}^{N} \alpha_{i, j}\right)}{2} \\
& \pm \frac{\sqrt{\left(N\left(\sum_{j=1, j \neq i}^{N} \alpha_{i, j}\right)\right)^{2}-4 k_{i}}}{2}, \\
& \operatorname{Re}(\lambda(A))<0 .
\end{aligned}
$$

The largest and the smallest eigenvalues of symmetric positive definite matrix $S$ are $\lambda_{\max }(S)$ and $\lambda_{\min }(S)$, respectively. The symmetric positive definite matrix $S$ with appropriate dimensions satisfies the following conclusion [41, 42].

$$
\lambda_{\min }(S)\left\|\zeta_{i}\right\|^{2} \leq \zeta_{i}^{T} S \zeta_{i} \leq \lambda_{\max }(S)\left\|\zeta_{i}\right\|^{2} .
$$

Finally, select Lyapunov function

$$
E_{i}=\zeta_{i}^{T} G \zeta_{i}, \quad G^{T}=G, \quad \lambda(G)>0 .
$$

Time derivative can be obtained:

$$
\begin{gathered}
\dot{E}_{i}=\dot{\zeta}_{i}^{T} G \zeta_{i}+\zeta_{i}^{T} G \dot{\zeta}_{i}=\left(A \zeta_{i}-B\right)^{T} G \zeta_{i}+\zeta_{i}^{T} G\left(A \zeta_{i}-B\right) \\
=-\zeta_{i}^{T}\left(A^{T} G+G A\right) \zeta_{i}-2 \zeta_{i}^{T} G B, \\
U_{i j}\left(\left\|p_{i}-p_{j}\right\|\right)\left\|p_{i}-p_{j}\right\| \leq l C .
\end{gathered}
$$

Therefore, according to the above formula we obtain

$$
\dot{E}_{i} \leq-\lambda_{\min }\left(A^{T} G+G A\right)\left\|\zeta_{i}\right\|^{2}+2 \lambda_{\max }(G)\left\|\zeta_{i}\right\|(N-1) l C .
$$

When

$$
\left\|\zeta_{i}\right\|>\frac{2 \lambda_{\max }(G)(N-1) l C}{\lambda_{\min }\left(A^{T} G+G A\right)}
$$

$\dot{E}_{i}<0$. The system continues to move closer to the population centre. Therefore, eventually the system stabilizes at a known system of

$$
\left\|\zeta_{i}\right\| \leq \frac{2 \lambda_{\max }(G)(N-1) l C}{\lambda_{\min }\left(A^{T} G+G A\right)}
$$

\section{Simulation of System Flocking Formation Behavior}

According to the UAV's physical characteristics, this paper will discretize the time with high frequency. Thus, a UAV $i$ makes its path decision $P_{i}(t+1)$ at time-step $t$ and will execute an action as the following equation:

$$
P_{i}(t+1)=P_{i}(t)+v \Delta t .
$$

The movement of the individual is not only controlled by itself but also affected by the state of other individuals.

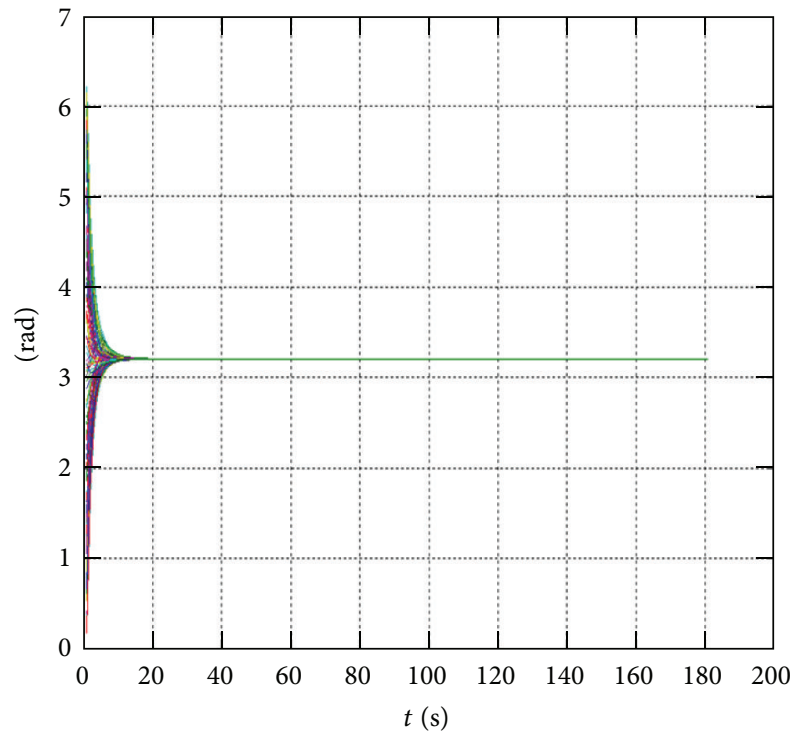

FIGURE 1: Velocities with respect to time.

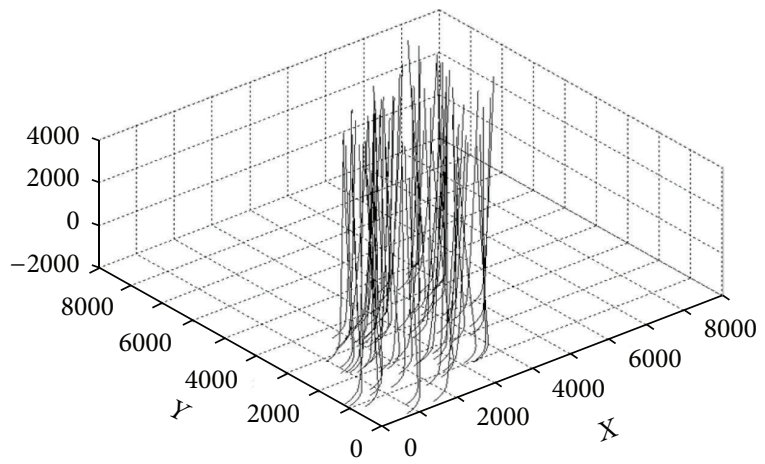

FIGURE 2: Trajectories with respect to time.

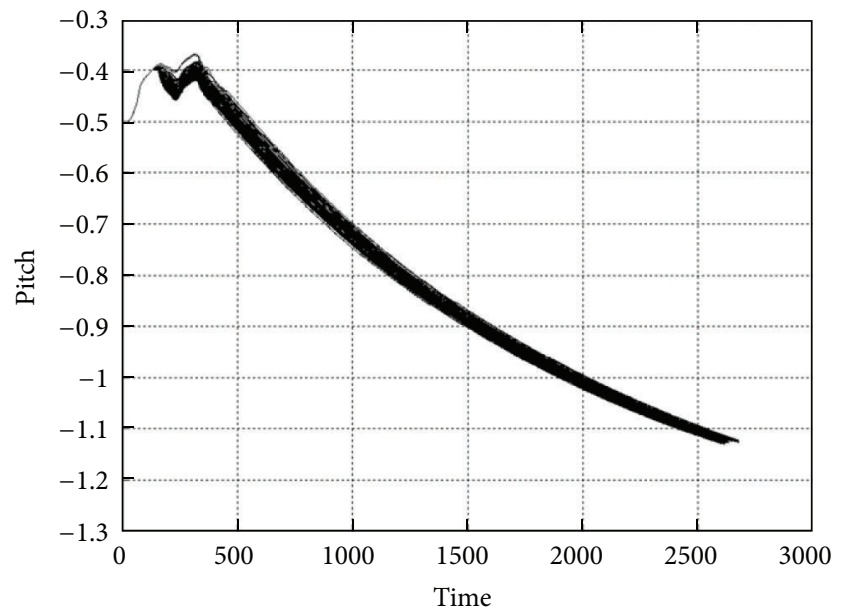

Figure 3: Angle of the Pitch with respect to time. 


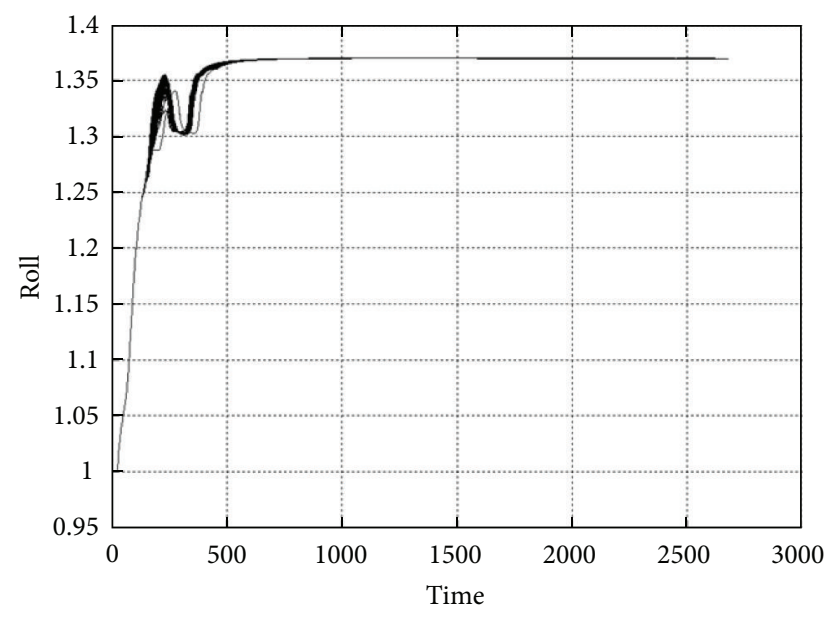

FIgUre 4: Angle of the Roll with respect to time.

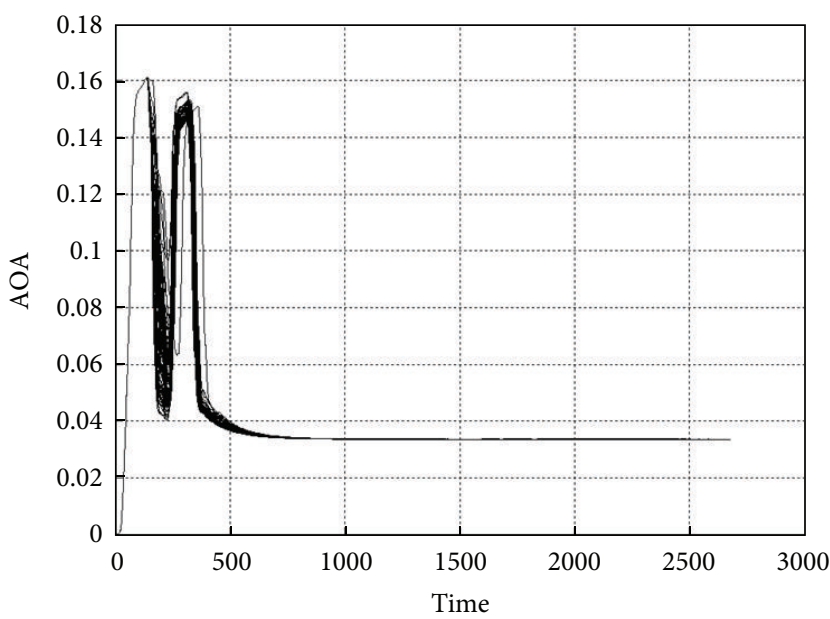

Figure 5: Angle of the Attack with respect to time.

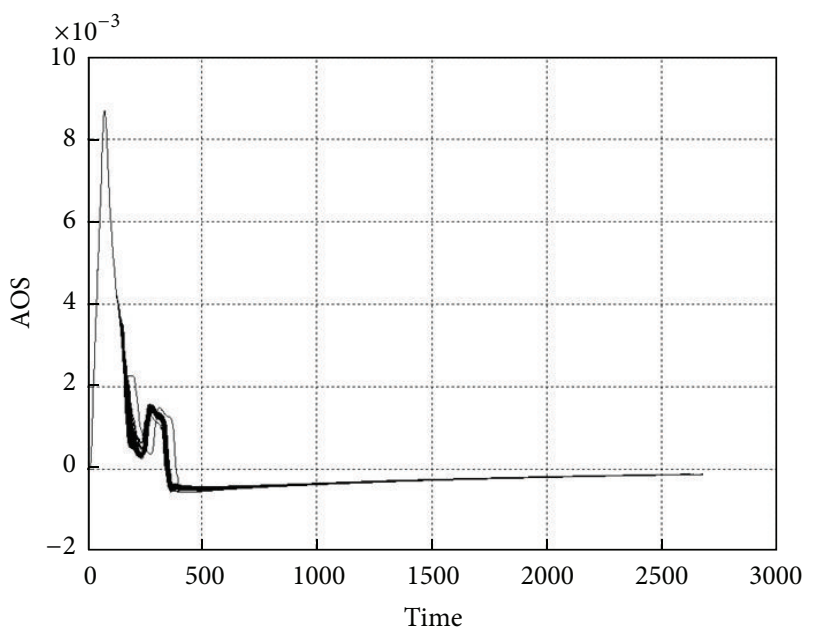

FIgURE 6: Angle of the Sideslip with respect to time.
Therefore, the individual direction of movement at a certain time is not only relative to its direction one moment before, but also relative to the directions of its surrounding individuals' movements. The influence of all the individuals to the individual $i$ can be described as the following equation:

$$
\frac{1}{\sum 1 / p_{i j}} \sum_{j=1}^{n} \frac{\theta_{j}(t)}{p_{i j}}
$$

Then, the speed direction of the UAV $i$ at time $(t+1)$ can be modified as the following equation:

$$
\theta_{i}(t+1)=\alpha \theta_{i}(t)+\beta \frac{1}{\sum 1 / p_{i j}} \sum_{j=1}^{n} \frac{\theta_{j}(t)}{p_{i j}}+\gamma \arctan \frac{y_{0}-y_{1}}{x_{0}-x_{1}}
$$

We consider the swarms of 100 UAVs with six degrees of freedom. The weights of the cost function are set to $\alpha=$ $0.3, \beta=0.5, \gamma=0.2, V=[200,0,0] \mathrm{m} / \mathrm{s}$ and $m=25 \mathrm{~kg}$. Direction is the rand variable from $-2 *$ pi to $2 *$ pi. The position of the UAVs is the rand variable. The factors of the influence on the flight are wind and airstream. The results for the case of 100 UAVs are shown in Figures 1 and 2.

From Figure 1, at $t=19 \mathrm{~s}$, the velocities of the swarms achieve consensus at $\theta=3.2$.

Figure 2 describes the trajectories with respect to time. The UAV swarms system will eventually be able to form a stable distance between each individual and the same velocity vectors. Collision between individuals is thus avoided.

Figures 3, 4, and 5 show the Pitch, Roll, and Attack with respect to time. From the simulation results, we can conclude that the UAVs based on the method successfully fly after the adjustment at the initial stage.

Figure 6 shows the Sideslip with respect to time. Through the analysis of the Sideslip Angle, we can find that the Angle of the Sideslip is less than 0.5 degrees and tends to zero, to ensure the turning flight control.

\section{Conclusion}

This paper analyzed current researches and existent problems of UAV swarms. Afterwards, by the theory of stochastic process and supplemented variables, a differential-integral model was established. The existence and uniqueness of the solution of the system were discussed. The flocking control law is given based on artificial potential with system consensus. At last, we analyzed the stability of the proposed flocking control algorithm based on the Lyapunov approach and proved the conclusion that the system in $28 \mathrm{~s}$ can converge to the consensus direction of the velocity. And we performed simulation tests to verify the conclusion.

\section{Acknowledgment}

This paper is supported by The National Defense PreResearch Foundation of China (Grant no. B222011XXXX). 


\section{References}

[1] H. B. Duan, Q. N. Luo, and G. J. Ma, "Hybrid particle swarm optimization and genetic algorithm for multi-UAV formation reconfiguration," IEEE Computational Intelligence Magazine, vol. 8, pp. 16-27, 2013.

[2] W. Yi, M. B. Blake, and R. G. Madey, "An operation-time simulation framework for UAV swarm configuration and mission planning," Procedia Computer Science, vol. 18, pp. 1949-1958, 2013.

[3] Y. Tang, H. J. Gao, J. Kurths, and J.-A. Fang, "Evolutionary pinning control and its application in UAV coordination," IEEE Transactions on Industrial Informatics, vol. 8, no. 4, pp. 828-838, 2012.

[4] G. B. Lamont, J. N. Slear, and K. Melendez, "UAV swarm mission planning and routing using multi-objective evolutionary algorithms," in Proceedings of the 1st IEEE Symposium of Computational Intelligence in Multicriteria Decision Making (MCDM '07), pp. 10-20, April 2007.

[5] E. Besada-Portas, L. de la Torre, J. M. de la Cruz, and B. de Andrés-Toro, "Evolutionary trajectory planner for multiple UAV in realistic scenarios," IEEE Transactions on Robotics, vol. 26, no. 4, pp. 619-634, 2010.

[6] S. Kanchanavally, R. Ordonez, and C. J. Schumacher, "Path planning in three dimensional environment using feedback linearization," in Proceedings of the American Control Conference (ACC '06), pp. 3545-3550, Mineapolis, Minn, USA, June 2006.

[7] M. Shanmugavel, A. Tsourdos, R. Zbikowski, and B. A. White, "3D path planning for multiple UAV s using pythagorean hodograph curves," in Proceedings of the AIAA Guidance, Navigation, and Control Conference and Exhibit, pp. 1576-1589, Hilton Head, SC, USA, August 2007.

[8] I. Hasircioglu, H. R. Topcuoglu, and M. Ermis, “3-D path planning for the navigation of unmanned aerial vehicles by using evolutionary algorithms," in Proceedings of the 10th Annual Genetic and Evolutionary Computation Conference (GECCO '08), pp. 1499-1506, July 2008.

[9] P. Vincent and I. Rubin, "A framework and analysis for cooperative search using UAV swarms," in Proceedings of the ACM Symposium on Applied Computing, pp. 79-86, 2004.

[10] G. Varela, P. Caamamño, F. Orjales, Á. Deibe, F. López-Peña, and R. J. Duro, "Swarm intelligence based approach for real time UAV team coordination in search operations," in Proceedings of the 3rd World Congress on Nature and Biologically Inspired Computing (NaBIC '11), pp. 365-370, October 2011.

[11] Y. 1. Yang, Cooperative search by uninhabited air vehicles in dynamic environment [Ph.D. thesis], University of Cincinnati, Cincinnati, Ohio, USA, 2005.

[12] P. Dasgupta, "A multiagent swarming system for distributed automatic target recognition using unmanned aerial vehicles," IEEE Transactions on Systems, Man, and Cybernetics A, vol. 38, no. 3, pp. 549-563, 2008.

[13] M. Yunhong, J. Zhe, and Z. Deyun, "A faster pruning optimization algorithm for task assignment," Journal of Northwestern Polytechnical University, vol. 31, pp. 40-43, 2013.

[14] B. Di, R. Zhou, and Q.-X. Ding, "Distributed coordinated heterogeneous task allocation for unmanned aerial vehicles," Control and Decision, vol. 28, pp. 274-278, 2013.

[15] W. You, Sh. Wang, and J. Tao, "Multi-UAV dynamic task assignment by ISODATA restrained clustering," Electronics Optics \& Control, vol. 17, pp. 22-26, 2010.
[16] D. Dionne and C. A. Rabbath, "Multi-UAV decentralized task allocation with intermittent communications: the DTC algorithm," in Proceedings of the American Control Conference (ACC '07), pp. 5406-5411, July 2007.

[17] P. Dasgupta and M. Hoeing, "Dynamic pricing algorithms for task allocation in multi-agent swarms," in Massively Multi-Agent Technology, N. Jamali, P. Scerri, and T. Sugawara, Eds., vol. 5043 of Lecture Notes in Computer Science, pp. 64-79, 2008.

[18] P. Gaudiano, B. Shargel, and E. Bonabeau, Swarm Intelligence: A New C2 Paradigm with an Application to Control Swarms of UAVs, Icosystem, Cambridge, Mass, USA, 2003.

[19] J. Finke, K. M. Passino, S. Ganapathy, and A. Sparks, "Modeling and analysis of cooperative control systems for uninhabited autonomous vehicles," in Cooperative Control, V. Kumar, N. Leonard, and A. S. Morse, Eds., vol. 309 of Lecture Notes in Control and Information Science, pp. 79-102, Springer, New York, NY, USA, 2005.

[20] T. McLain, R. Beard, and J. Kelsey, "Experimental demonstration of multiple robot cooperative target intercept," in Proceedings of the AIAA Guidance, Navigation, and Control Conference, AIAA-2002-4678, 2002.

[21] A. Moitra, R. Szczerba, V. Didomizio, L. Hoebel, R. Mattheyses, and B. Yamrom, "A novel approach for the coordination of multi-vehicle teams," in Proceedings of the AIAA Guidance, Navigation, and Control Conference, pp. 608-618, Monterey, Calif, USA, 2001.

[22] P. Vincent and I. Rubin, "A framework and analysis for cooperative search using UAV swarms," in Proceedings of the ACM Applied Computing, pp. 79-86, Nicosia, Cyprus, 2004.

[23] H. Hexmoor, B. McLaughlan, and M. Baker, "Swarm control in unmanned aerial vehicles," in Proceedings of the International Conference on Artificial Intelligence (ICAI '05), pp. 911-917, June 2005.

[24] R. Garcia and L. Barnes, "Multi-UAV simulator utilizing xplane," Journal of Intelligent and Robotic Systems, vol. 57, no. 1-4, pp. 393-406, 2010.

[25] M. A. Russell, G. B. Lamont, and K. Melendez, "On using SPEEDES as a platform for a parallel swarm simulation," in Proceedings of the Winter Simulation Conference, pp. 1129-1137, December 2005.

[26] S. Luke, C. Cioffi-Revilla, L. Panait, K. Sullivan, and G. Balan, "MASON: a multiagent simulation environment," Simulation, vol. 81, no. 7, pp. 517-527, 2005.

[27] S. J. Rasmussen, J. W. Mitchell, P. R. Chandler, C. J. Schumacher, and A. L. Smith, "Introduction to the Multi-UAV2 simulation and its application to cooperative control research", in Proceedings of the American Control Conference (ACC '05), pp. 44904501, June 2005.

[28] T. McLain, R. Beard, and J. Kelsey, "Experimental demonstration of multiple robot cooperative target intercept," in Proceedings of the AIAA Guidance, Navigation, and Control Conference, AIAA-2002-4678, Monterey, Calif, USA, 2002.

[29] H. G. Tanner, A. Jadbabaie, and G. J. Pappas, "Stable flocking of mobile agents, part I: fixed topology," in Proceedings of the 42nd IEEE Conference on Decision and Control, pp. 2010-2015, December 2003.

[30] H. G. Tanner, A. Jadbabaie, and G. J. Pappas, "Stable flocking of mobile agents part II: dynamic topology," in Proceedings of the 42nd IEEE Conference on Decision and Control, pp. 2016-2021, December 2003. 
[31] M. J. Mataric, Interaction and intelligent behavior [Ph.D. thesis], Massachusetts Institute of Technology, Cambridge, Mass, USA, 1994.

[32] I. Kelly and D. Keating, "Flocking by the fusion of sonar and active infrared sensors on physical autonomous robots," in Proceedings of the Conference on Mechatronics and Machine Vision in Practice, pp. 14-17, 1996.

[33] A. T. Hayes and P. Dormiani-Tabatabaei, "Self-organized flocking with agent failure: off-line optimization and demonstration with real robots," in Proceedings of the IEEE International Conference on Robotics and Automation, pp. 3900-3905, May 2002.

[34] O. Holland, J. Woods, R. de Nardi, and A. Clark, "Beyond swarm intelligence: the ultraswarm," in Proceedings of the IEEE Swarm Intelligence Symposium (SIS '05), pp. 217-224, June 2005.

[35] E. Ferrante, A. E. Turgut, N. Mathews, M. Birattari, and M. Dorigo, "Flocking in stationary and non-stationary environments: a novel communication strategy for heading alignment," in Parallel Problem Solving from Nature-PPSN XI, R. Schaefer, C. Cotta, J. Kolodziej, and G. Rudolph, Eds., vol. 6239 of Lecture Notes in Computer Science, pp. 331-340, 2010.

[36] A. Stranieri, E. Ferrante, A. E. Turgut et al., "Self-organized flocking with a heterogeneous mobile robot swarm," Tech. Rep., 2011.

[37] B. Li, "Stochastic process model of the multi-UAV s collaborative system based on state transition," in Proceedings of Conference on Modeling, Identification and Control, pp. 757-761, 2012.

[38] Z. X. Chen, Partial Differential Equations, Science Press, Beijing, China, 2002.

[39] L. Guo, H. Xu, C. Gao, and G. Zhu, "Stability analysis of a new kind series system," IMA Journal of Applied Mathematics, vol. 75, no. 3, pp. 439-460, 2010.

[40] D. p. Gaver, "Time to failure and availability of paralleled system with repair," IEEE Transactions on Reliability, vol. 12, pp. 30-38, 1963.

[41] Q. J. Fan, Key techniques research of cooperative formation biomimetic flight control for multi-UAV [Ph.D. thesis], Nanjing University of Aeronautics and Astronautics, Nanjing, China, 2008.

[42] C. Yancai, Research on distributed cooperative control for swarm UAVs [Ph.D. thesis], Nanjing University of Aeronautics and Astronautics, Nanjing, China, 2011. 


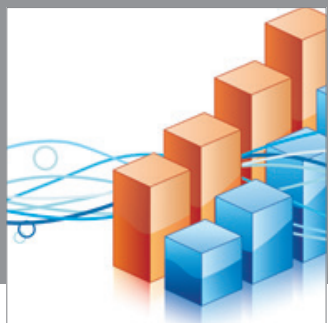

Advances in

Operations Research

mansans

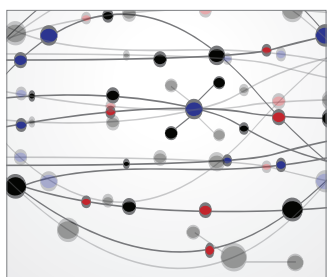

The Scientific World Journal
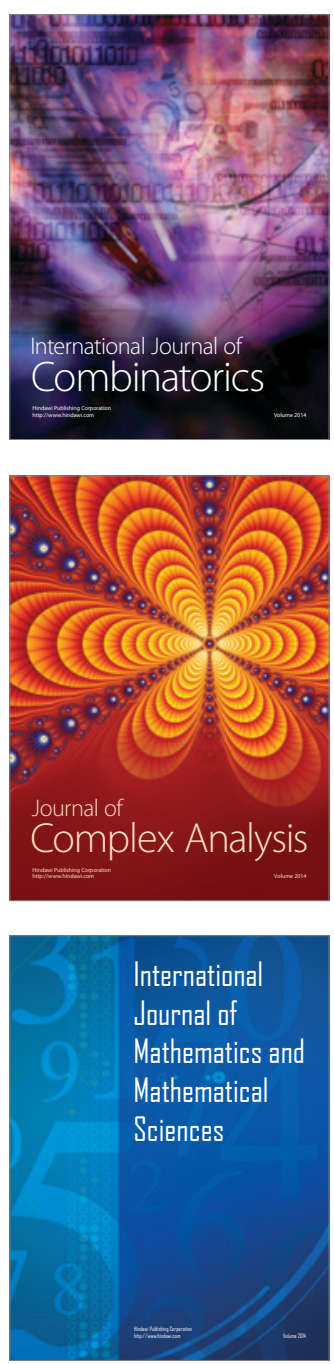
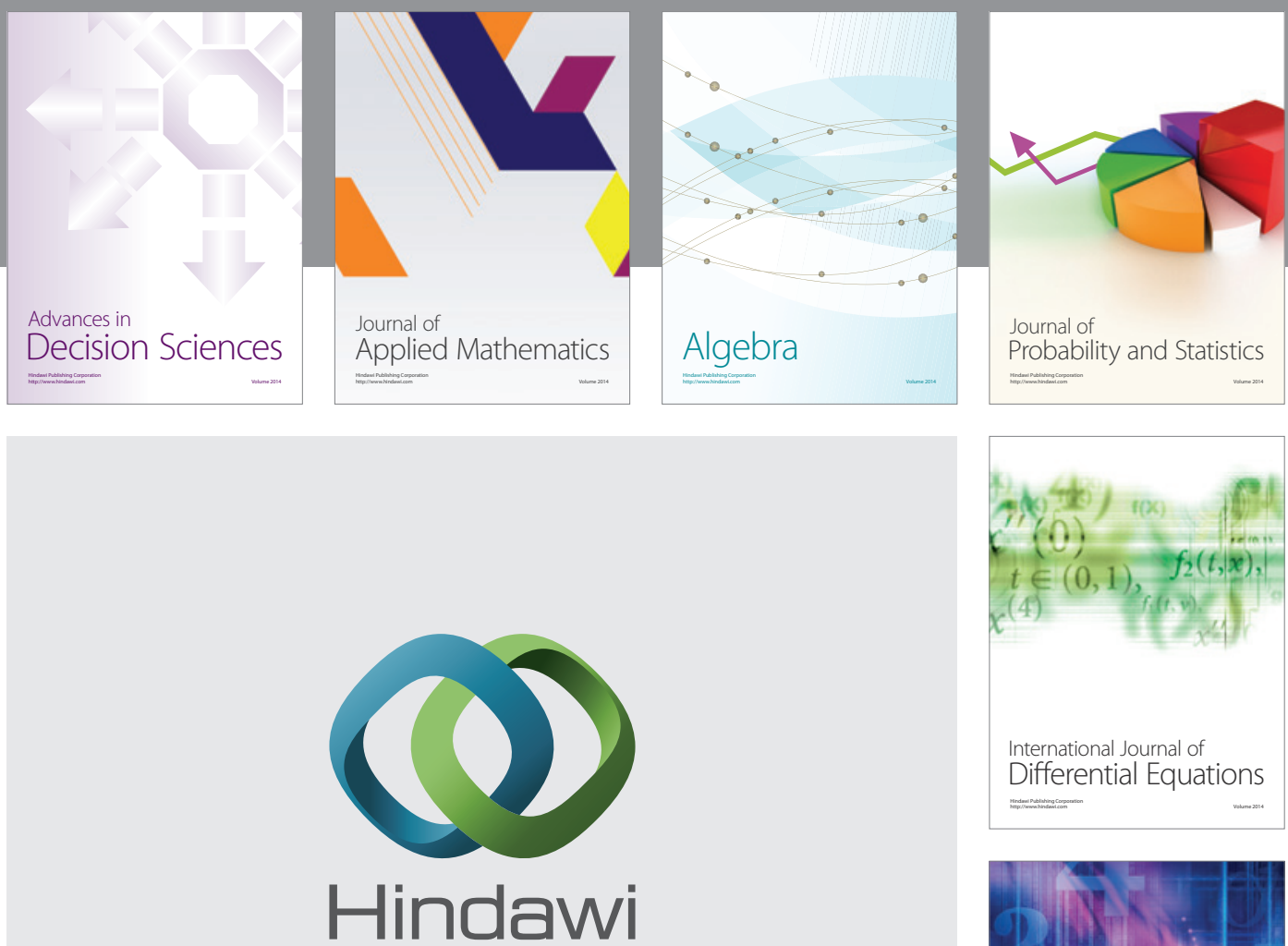

Submit your manuscripts at http://www.hindawi.com
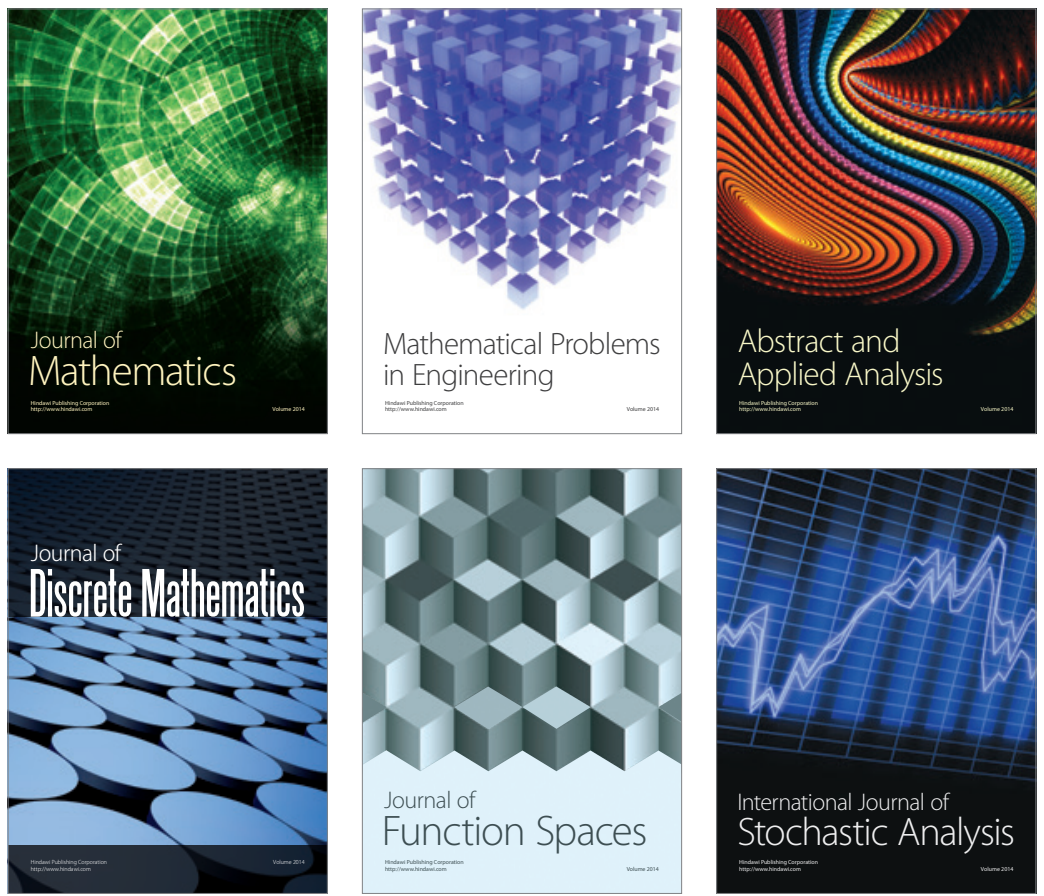

Journal of

Function Spaces

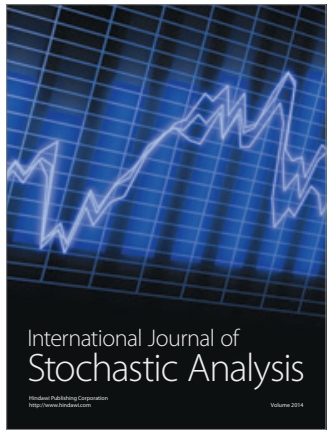

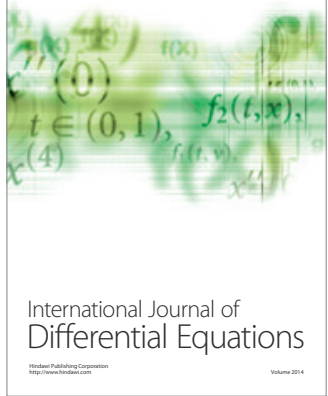
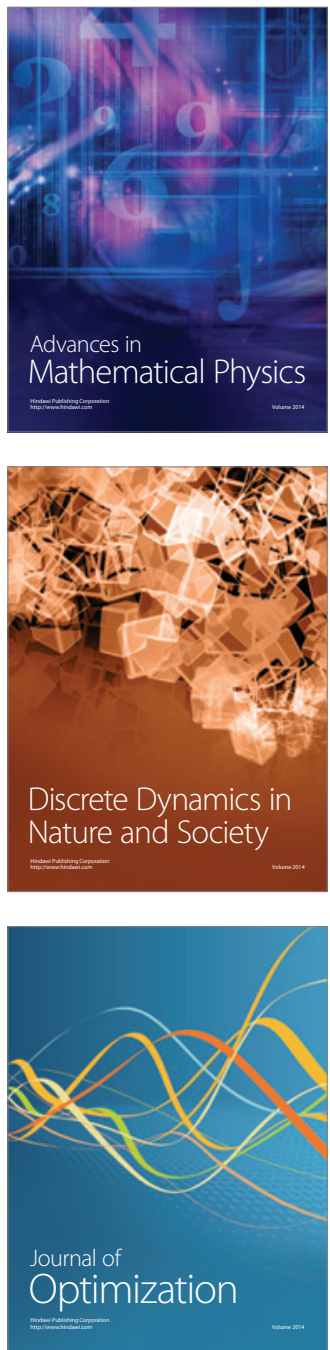